\section{International Scientific Journal Theoretical \& Applied Science}

\author{
p-ISSN: 2308-4944 (print) e-ISSN: 2409-0085 (online) \\ Year: 2014 Issue: 12 Volume: 20 \\ Published: $30.12 .2014 \quad$ http://www.T-Science.org
}

SECTION 5. Innovative technologies in science.
Andrey Mastislavovich Korneev candidate Technical Sciences, teacher at the University

Lipetsk State Technical University, Russia weenrok@mail.ru

Faisal Abdo Ali Al-Saeedi

Postgraduate, Postgraduate(student) Lipetsk State Technical University, Russia faisal853450@mail.ru

Ghassan Mohsen Al-Sabry

Postgraduate, Postgraduate(student) Lipetsk State Technical University, Russia lion100@mail.ru

Tatiana Andreevna Smetannikova

Postgraduate, Postgraduate(student) Lipetsk State Pedagogical University, Russia aveenrok@mail.ru

Abdullh Mohammed Mohammed Nagi

Postgraduate, Postgraduate(student) Lipetsk State Technical University, Russia nagi_farad@mail.ru

\title{
MODELING OF COMPLEX TECHNOLOGICAL PROCESSES VIA POLYNOMIAL ZHEGALKIN
}

Abstract: The work presented an algorithm for constructing polynomial Zhegalkin for arbitrary data sets with the original data in a number system different from binary. Shown principle of the algorithm and operation procedures of automatic processing of a specific example of a small data set consisting of positive integers.

Key words: Modeling, Modeling of complex technological processes, complex technological processes, polynomial Zhegalkin.

Language: Russian

Citation: Korneev AM, Al-Saeedi FA, Al-Sabry GM, Smetannikova TA, Nagi AM (2014) MODELING OF COMPLEX TECHNOLOGICAL PROCESSES VIA POLYNOMIAL ZHEGALKIN. ISJ Theoretical \& Applied Science 12 (20): 90-93. doi: http://dx.doi.org/10.15863/TAS.2014.12.20.19

\section{МОДЕЛИРОВАНИЕ СЛОЖНЫХ ТЕХНОЛОГИЧЕСКИХ ПРОЦЕССОВ С ПОМОЩЬЮ МНОГОЧЛЕНА ЖЕГАЛКИНА}

Аннотация: В работе представлен алгоритм построения полинома Жегалкина для произвольных массивов данных, с исходными данными в системе счисления, отличной от двоичной. Показан принции действия алгоритма и работа процедуры автоматической обработки на конкретном примере небольшого массива данных, состоящего из целых положительных чисел.

Ключевые слова: Моделирование, Моделирование сложных технологчческих прочессов, сложные технологические прочессы, многочлен Жегалкина.

Способ исследования технологии, использующий разбиение области, предлагается в работах [1-10] и предназначен для выбора и моделирования оптимальных технологических режимов. Методика предполагает поиск и отбор технологических траекторий, обеспечивающих с максимальной вероятностью получение продукции задаваемого качества. Технологическая траектория - это совокупность алфавитов исследуемых факторов технологии. Разбиение алфавитов в простейшем случае производится на равные интервалы по каждому из факторов, либо размеры элементов алфавитов выбираются исследователем.

Разбиение каждого интервала принадлежности будет осуществляться на равные участки, где количество участков определено правилом:

- если в числе, определяющем длину рассматриваемого интервала, один десятичный разряд, то количество интервалов разбиения задать равным длине интервала; 
- если в числе, определяющем длину рассматриваемого интервала, два десятичных разряда и длина интервала меньше 20 - количество интервалов определить по формуле:

$\left\lceil\frac{\text { длина интерваларазбиения }}{3}\right\rceil$, где \lceil\rceil - округление вверх до ближайшего целого;

- если в числе, определяющем длину рассматриваемого интервала, два десятичных разряда и длина интервала больше 20, но меньше 40 - количество интервалов определить по формуле:

$D_{\text {стари. }}+\left\lfloor D_{\text {стари. }} \cdot 0,25 \cdot\left(N_{\text {дес.рязр. }}+1\right) !\right\rfloor$,

где $D_{\text {стари. }}$ - старший десятичный разряд числа, определяющего длину рассматриваемого интервала, $N_{\text {дес.разр. }}{ }^{-}$ число десятичных разрядов в числе, определяющем длину рассматриваемого интервала,

L 」 - операция округления вниз до ближайшего целого;

- если в числе, определяющем длину рассматриваемого интервала, два десятичных разряда и длина интервала больше 40 - количество интервалов определить по формуле:

$$
D_{\text {стари. }}+\left\lfloor D_{\text {стари. }} \cdot 0,25 \cdot N_{\text {дес.рязр. }}\right\rfloor,
$$

где обозначения аналогичными, описанным выше.

Моделировать зависимость сочетаний алфавитов выходов от сочетания алфавитов входов можно с использованием многочлена Жегалкина. После определения количества интервалов разбиения определяется шаг разбиения интервалов и границы каждого интервала.Каждому интервалу присваивается номер от 1 до $p$. Определяется, какому интервалу принадлежит каждый $x_{i}, y_{j}$ и строится таблица сочетаний, которая получается из исходной путем замены соответствующего элемента на номер интервала, в который он попадает.Определение необходимого для двоичного представления таблицы сочетаний числа двоичных разрядов по каждому столбцу таблицы сочетаний. Необходимое число разрядов определяется следующим образом: определяется максимальный элемент по столбцу; из него вычитается единица; полученный результат преобразуется в двоичную систему счисления; минимальное число двоичных разрядов, достаточное для представления числа, полученного на предыдущем шаге и будет являться искомым числом.

Двоичное кодирование таблицы сочетаний, в результате которого каждый столбец разбивается на несколько новых. Количество таких новых столбцов определяется результатом, полученным на предыдущем шаге. По набору столбцов, полученных из $x_{j}$ и одному столбцу из полученных по $y_{k}$, путем последовательного перебора $y_{k}$ строится полином Жегалкина. Для отсутствующих в двоичной таблице сочетаний соответствующий коэффициент полинома Жегалкина принимается равным 0. При наличии одинаковых сочетаний по $\mathrm{x}_{\mathrm{j}}$, но различных по $y_{k}$, в соответствующий двоичный разряд $y_{k}$ записываем наиболее часто встречающийся результат.

Пример сочетания алфавитов приведен ниже.

\section{Сочетание алфавитов входов и выхода.}

Таблица 1

\begin{tabular}{|r|r|r|r|}
\hline $\mathrm{c} 1$ & $\mathrm{c} 2$ & $\mathrm{c} 3$ & $\mathrm{e} 1$ \\
\hline 1 & 1 & 1 & 1 \\
\hline 1 & 2 & 1 & 1 \\
\hline 1 & 1 & 3 & 1 \\
\hline 1 & 2 & 3 & 1 \\
\hline 1 & 1 & 1 & 3 \\
\hline 1 & 1 & 2 & 3 \\
\hline 1 & 3 & 1 & 3 \\
\hline 1 & 1 & 1 & 3 \\
\hline 1 & 2 & 2 & 3 \\
\hline 1 & 3 & 3 & 1 \\
\hline 1 & 1 & 1 & 1 \\
\hline 1 & 1 & 2 & 2 \\
\hline
\end{tabular}

ISPC European Research, 


\begin{tabular}{|r|r|r|r|}
\hline 1 & 1 & 1 & 1 \\
\hline 1 & 3 & 1 & 2 \\
\hline 1 & 1 & 2 & 2 \\
\hline 1 & 1 & 3 & 3 \\
\hline 1 & 1 & 1 & 2 \\
\hline 1 & 1 & 3 & 1 \\
\hline 1 & 1 & 3 & 3 \\
\hline 1 & 2 & 1 & 2 \\
\hline 1 & 3 & 2 & 1 \\
\hline 1 & 1 & 1 & 1 \\
\hline
\end{tabular}

где $c 1, c 2, c 3$ - входы, а e1 - выход. Применим к данному массиву процедуру обработки, написанную по описанному выше алгоритму.

Далее определено необходимое для двоичного представления каждого столбца таблицы число двоичных разрядов. На следующем этапе строится двоичная таблица сочетаний.

Двоичное представление сочетаний алфавитов входов и выхода.

Таблица 2

\begin{tabular}{|r|r|r|r|r|r|r|}
\hline \multicolumn{2}{|c|}{$\mathrm{c} 3$} & \multicolumn{2}{c|}{$\mathrm{c} 2$} & $\mathrm{c} 1$ & \multicolumn{2}{c|}{$\mathrm{e} 1$} \\
\hline 0 & 0 & 0 & 0 & 0 & 0 & 0 \\
\hline 0 & 0 & 0 & 1 & 0 & 0 & 0 \\
\hline 1 & 0 & 0 & 0 & 0 & 0 & 0 \\
\hline 1 & 0 & 0 & 1 & 0 & 0 & 0 \\
\hline 0 & 0 & 0 & 0 & 0 & 1 & 0 \\
\hline 0 & 1 & 0 & 0 & 0 & 1 & 0 \\
\hline 0 & 0 & 1 & 0 & 0 & 1 & 0 \\
\hline 0 & 0 & 0 & 0 & 0 & 1 & 0 \\
\hline 0 & 1 & 0 & 1 & 0 & 1 & 0 \\
\hline 1 & 0 & 1 & 0 & 0 & 0 & 0 \\
\hline 0 & 0 & 0 & 0 & 0 & 0 & 0 \\
\hline 0 & 1 & 0 & 0 & 0 & 0 & 1 \\
\hline 0 & 0 & 0 & 0 & 0 & 0 & 0 \\
\hline 0 & 0 & 1 & 0 & 0 & 0 & 1 \\
\hline 0 & 1 & 0 & 0 & 0 & 0 & 1 \\
\hline 1 & 0 & 0 & 0 & 0 & 1 & 0 \\
\hline 0 & 0 & 0 & 0 & 0 & 0 & 1 \\
\hline 1 & 0 & 0 & 0 & 0 & 0 & 0 \\
\hline 1 & 0 & 0 & 0 & 0 & 1 & 0 \\
\hline 0 & 0 & 0 & 1 & 0 & 0 & 1 \\
\hline 0 & 1 & 1 & 0 & 0 & 0 & 0 \\
\hline 0 & 0 & 0 & 0 & 0 & 0 & 0 \\
\hline & & & & & & \\
\hline 0
\end{tabular}

В полученной таблице сочетаний есть такие сочетания $c 1, c 2, c 3$, которым соответствуют несколько значений $e 1$. При построении таблицы всех возможных двоичных сочетаний значения в столбцах, соответствующих двоичному представлению el будем использовать наиболее часто встречающиеся в исходной двоичной таблице сочетаний.
Произведем построение полинома Жегалкина, согласно алгоритму по полученной выше таблице.

Первый столбец $e 1$ :

$$
\begin{array}{ccc}
\mathrm{a}_{0}=0 ; & \mathrm{a}_{1}=0 ; & \mathrm{a}_{2}=0 ; \\
\mathrm{a}_{3}=1 ; & \mathrm{a}_{4}=0 ; & \mathrm{a}_{5}=1 ; \\
\mathrm{a}_{42}=1 ; & \mathrm{a}_{43}=1 ; & \mathrm{a}_{52}=1 ; \\
\mathrm{a}_{53}=1 . & & \\
P_{1}=a_{3} c_{3} \oplus a_{42} c_{4} c_{2} \oplus a_{43} c_{4} c_{3} \oplus a_{53} c_{5} c_{3} .
\end{array}
$$


Второй столбец $e 1$ :

$\mathrm{a}_{0}=0 ; \quad \mathrm{a}_{1}=0 ; \quad \mathrm{a}_{2}=0 ; \quad \mathrm{a}_{3}=0 ; \quad \mathrm{a}_{4}=1 ; \mathrm{a}_{5}=0 ;$

$\mathrm{a}_{42}=1 ; \quad \mathrm{a}_{43}=1 ; \quad \mathrm{a}_{52}=0 ; \quad \mathrm{a}_{53}=0$.
$P_{2}=a_{4} c_{4} \oplus a_{42} c_{4} c_{2} \oplus a_{43} c_{4} c_{3}$.

В итоге конечный результат будет иметь вид:

$$
P=\left(a_{3} c_{3} \oplus a_{42} c_{4} c_{2} \oplus a_{43} c_{4} c_{3} \oplus a_{53} c_{5} c_{3}\right) \&\left(a_{4} c_{4} \oplus a_{42} c_{4} c_{2} \oplus a_{43} c_{4} c_{3}\right)
$$

\section{Заключение}

Показан алгоритм построения полинома Жегалкина для произвольных массивов данных, с исходными данными в системе счисления, отличной от двоичной. На языке VBA реализована процедура автоматической обработки входного массива для дальнейшего построения по нему полинома Жегалкина. Показан принцип действия алгоритма и работа процедуры автоматической обработки на конкретном примере небольшого массива данных, состоящего из целых положительных чисел.

\section{References:}

1. AM Korneev, FA Al-Saeedi, GM Al-Sabry, AM Nagi (2014) Blocks of structural modeling and search optimization discrete cellhierarchical systems using computer information processing techniques // International Scientific Journal, Theoretical \& Applied Science. «Modern mathematics in Science» Caracas, Venezuela - № 6 (14), 2014, pp. 14 -17. doi: http://dx.doi.org/10.15863/TAS.2014.06.14.4

2. Korneev AM, Butakov VV, Filatov AA (2014) Discrete-variable modeling of technological process // International Scientific Journal, Theoretical \& Applied Science. «Scientific technologies of the future», Linköping, Sweden. - №2, 2014. pp. 35-39. doi: http://dx.doi.org/10.15863/TAS.2014.02.10.5

3. Korneev AM, Al-Saeedi FA, Al-Sabry GM, Smetannikova TA, Nagi AM (2014) Discrete modeling of complex manufacturing systems // International Scientific Journal, Theoretical \& Applied Science. «Economy, technology, education and prospects for 2014», Malmö, Sweden. - №1, 2014. pp. 32-35. doi: http://dx.doi.org/10.15863/TAS.2014.01.9.5

4. Korneev AM, Al-Sabry GM, Al-Saeedi FA (2013) The optimal strategy for adapting technological regimes in discrete systems // Proceedings of the 4rd International Academic Conference «Applied and Fundamental Studies» Vol. I ,St. Louis, Missouri, USA . 2013, pp. 264-267.

5. Korneev AM, Blyumin SL, Smetannikova TA (2013) Chislennye metody poiskovoy optimizatsii diskretnykh kletochnoierarkhicheskikh sistem [Tekst] / Korneev A.M., Blyumin S.L., Smetannikova T.A. // Vesti vysshikh uchebnykh zavedeniy Chernozem'ya. - 2013. - №3. - pp. 21-26.

6. Korneev AM, Al-Saeedi FA, Al-Sabry GM, Nagi AM (2014) The analysis of technological trajectories based on the tree construction // International Scientific Journal, Theoretical \& Applied Science. «European Innovation», Martigues, France - № 9 (17), 2014, pp. 46-49. doi: http://dx.doi.org/10.15863/TAS.2014.09.17.7

7. (2008) Opisanie tekhnologiy s pomoshch'yu konechnykh avtomatov Korneev A.M. Vesti vysshikh uchebnykh zavedeniy Chernozem'ya. 2008. № 3. pp. 56-61.

8. Korneev AM, Miroshnikova TV (2008) Metodika poiska optimal'nykh granits faktorov skvoznoy tekhnologii. Sistemy upravleniya i informatsionnye tekhnologii. 2008, №3(33), pp.93-96.

9. Korneev AM (2009) Metody identifikatsii skvoznoy tekhnologii proizvodstva metalloproduktsii [Tekst]: monografiya / A.M. Korneev; Lipetskiy gosudarstvennyy pedagogicheskiy universitet. - Lipetsk: LGPU, 2009. - 286.

10. Blyumin SL, Korneev AM (2005) Diskretnoe modelirovanie sistem avtomatizatsii i upravleniya [Tekst]: Monografiya; Lipetskiy ekologo-gumanitarnyy institut. - Lipetsk: LEGI, 2005, - 124. 